\title{
Performance Analysis of X-Ray Phase-Contrast Interferometers with Respect to Grating Layouts
}

\author{
Wilhelm Haas ${ }^{a, b}$, P. Bartl ${ }^{b}$, F. Bayer ${ }^{b}$, J. Durst ${ }^{b}$, T. Grund ${ }^{c}$, J. Kenntner ${ }^{c}$, T. Michel ${ }^{b}$,

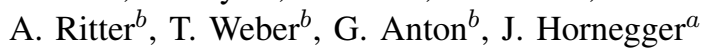 \\ ${ }^{a}$ Pattern Recognition Lab, University of Erlangen-Nuremberg \\ ${ }^{b}$ ECAP Novel Detectors / Medical Physics, University of Erlangen-Nuremberg \\ ${ }^{c}$ Institute for Microstructure Technology, Karlsruhe Institute of Technology (KIT)
}

\begin{abstract}
The grating-based phase-contrast imaging approach is highly dependent on the quality of the used gratings. While the fabrication of gratings for the soft $\mathrm{X}$-ray range is more or less well controllable, the fabrication for the hard $X$-ray range $(>30 \mathrm{keV})$ is more challenging as the gratings must have high aspect ratios and thus fine structures. One of the best fabrication technologies for such gratings is LIGA (Lithography, Electroplating and Molding). However, due to such small structures and high aspect ratio it is unavoidable that the gratings become nonperfect and have deformations. Since the fabrication is complex, expensive and time consuming, a simple way is needed to assess the influence of such deformations on the signal and also a simple way to design and test new grating layouts.

This work presents a simulation framework for $\mathrm{X}$-ray phasecontrast imaging which allows to model, simulate and assess the quality of arbitrary grating layouts in an easier, cheaper and faster way. Furthermore, it allows the assessment of the quality of new grating layouts as well as of existing gratings.
\end{abstract}

\section{INTRODUCTION}

$\mathbf{P}$ HASE-contrast imaging with X-rays is an approach which is used to gain information about the real part of the complex refractive index of a material. This work focuses on the grating-based interferometer [1], [2] which enables the measurement of both components of the complex refractive index. As its name suggests, the system is based on gratings. Thus, it is highly dependent on the quality of the used gratings. The challenging part is the fabrication of analyzer gratings for the hard X-ray range ( $>30 \mathrm{keV})$ as they require a highly absorbing material and high aspect ratio ( $>50)$. Only the LIGA fabrication approach is able to produce gratings with such a high aspect ratio. However, difficulties in the fabrication process cause deformations of the grating structure and thus a deviation from the theoretically ideal grating. In order to investigate the effects of these deformations one can produce many different gratings which is complex, expensive and time consuming. An easier, cheaper and faster way is to simulate such layouts numerically.

\section{GRATingS - LIGA}

LIGA stands for the German acronym for Lithographie, Galvanoformung and Abformung (Lithography, Electroplating and Molding) [3]. It is one of the best fabrication technologies to fabricate gratings with the required aspect ratio for hard a)
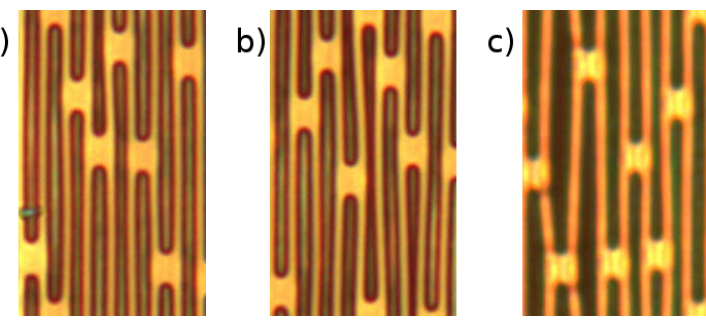

Fig. 1. Cutouts of some optical light microscope images of gratings with a) no deformations; b) wavy and too thin bars; c) too thick bars

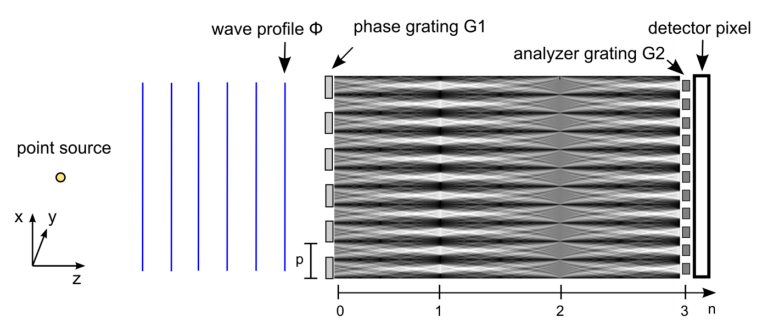

Fig. 2. Basic setup of a grating-based interferometer with the simulated intensity distribution between phase grating and analyzer grating.

X-ray grating-based phase-contrast imaging. Fig. 1a shows a cutout of an analyzer grating designed and fabricated at the Institute for Microstructure Technology, Karlsruhe Institute of Technology (KIT) [3]. The bright parts correspond to the low absorbing material (SU-8) and darker ones to high absorbing material $(\mathrm{Au})$. The gaps between the gold bars stabilize the structure otherwise the walls would collapse. On the other hand fig. $1 \mathrm{~b}$ and $1 \mathrm{c}$ represent analyzer gratings with deformed bars where some of them are too thick, too thin or wavy. Such deformations change the local periodicity and duty cycle of the gratings, hence, have an affect on the interferometry.

\section{Simulation}

A basic setup of a grating-based phase-contrast imaging system is shown in fig. 2. Generally three computation steps have to be performed. Firstly, transmission of the X-ray wave through the phase grating G1. Secondly, computation of the interference pattern downstream towards the analyzer grating G2 and finally transmission through G2. Since only the 
a)

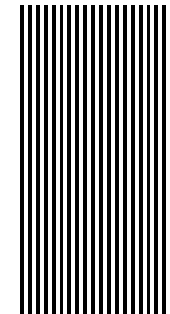

d)

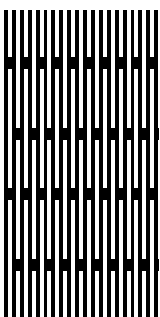

b)

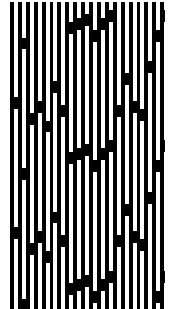

c)

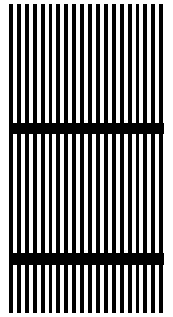

e)

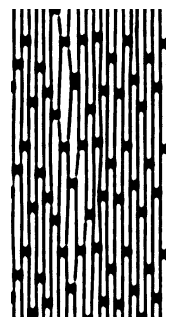

Fig. 3. Used phase and analyzer grating layouts to assess the influences Gratings a) to d) are geometrically ideal phantom gratings whereas b) to d) contain an equal number of stabilization gaps; b) randomly distributed gap positions; c) gaps aligned in rows; d) gaps in even columns are shifted by half of the bar length. The last grating layout e) is a binarized image of an existing analyzer grating acquired with an optical light microscope.

influence of the gratings is of interest the case with an object in path of the X-ray beams will not be considered, hence, the simulated plane or spherical wave starts right before G1. The first and third step is a transmission of the wave through the gratings. This is done by thin-sample approximation [5] of the gratings. The information about the grating structure can be gained e. g. from optical light microscope images. A much better source of information is a radiogram of the gratings which additionally contains information about local material heights. The second step - free space propagation from $\mathrm{G} 1$ to an arbitrary location e. g. grating G2 - is described by the solution of the Helmholtz equation in free space. The computation of the interference pattern downstream to a certain distance $d$ is given by a convolution of the wave function $\Phi$ with the Fresnel-Propagator $P_{\lambda}$ [5], [6]:

$$
\begin{gathered}
\Phi_{\lambda}(x, y, z=d)=\Phi_{\lambda}(x, y, z=0) * P_{\lambda}(x, y, d) \\
P_{\lambda}(x, y, d)=\frac{i k}{2 \pi d} \cdot \exp (i k d) \cdot \exp \left[\frac{i k\left(x^{2}+y^{2}\right)}{2 d}\right]
\end{gathered}
$$

where $k$ denotes the wavenumber and $\lambda$ the wavelength.

The finite focal spot size can be taken into account by a convolution of the interference pattern with the intensity distribution of the focal spot of the X-ray tube.

\section{RESUlts}

Following assumptions were made:

- General setup

- G1-G2 distance $0.09 m$ (first Talbot distance)

- $80 \mathrm{kV}$ tungsten spectrum, $1 \mathrm{~mm} \mathrm{Al}$ and $1 \mathrm{~mm} \mathrm{Si}$ filtering

- plane wave

- ideal detector

- Phase grating G1

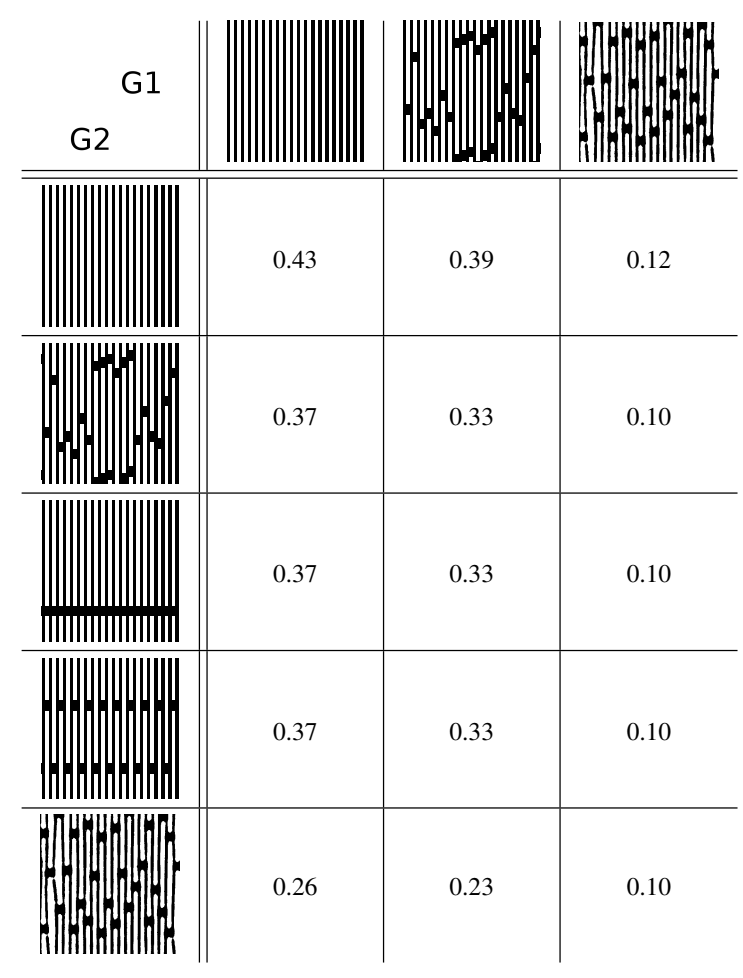

TABLE I

RESULTING VISIBILITIES COMPUTED AND SIMULATED BY THE PRESENTED FRAMEWORK FOR DIFFERENT COMBINATIONS OF GRATING LAYOUTS.

- material: $\mathrm{Ni}$

- periodicity: $4.8 \mu \mathrm{m}$

- duty cycle: 0.5

- height: $14.1 \mu \mathrm{m}$

- design energy: $40 \mathrm{keV}$

- Analyzer grating G2

- low absorbing material: SU-8

- high absorbing material: Au

- periodicity: $2.4 \mu \mathrm{m}$

- duty cycle: 0.5

- height: $120 \mu \mathrm{m}$

Fig. 3 displays some cutouts of the grating layouts used for the phase and the analyzer grating in these simulations. In case of a phase grating the bright areas correspond to the material $\mathrm{Ni}$ and dark areas to air. Used as analyzer grating layout the bright areas correspond to gold $(\mathrm{Au})$ and dark areas to SU-8. Fig. 3a illustrates a geometrically ideal phantom grating with continuous bars. Gratings fig. $3 \mathrm{~b}$ to fig. $3 \mathrm{~d}$ are also geometrically ideal gratings but with stabilization gaps between the bars. Each bar has a length of $30 \mu \mathrm{m}$ and each gap a length of $3 \mu \mathrm{m}$. The difference between these layouts is the distribution of the gaps. In fig. 3b the gap positions are randomly distributed, in fig. $3 c$ aligned in rows and in fig. $3 d$ each even numbered column is shifted by half of the bar length. Last grating layout fig. 3e is a binarized image of an existing analyzer grating taken by an optical light microscope. 


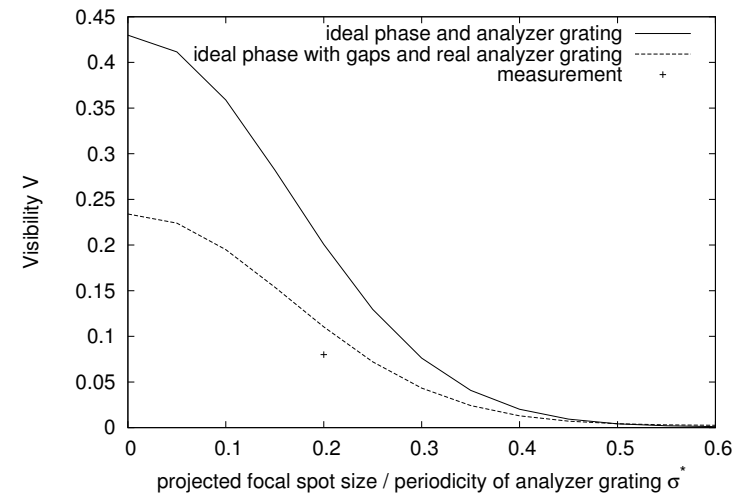

Fig. 4. Visibility plot with respect to the normalized projected focal spot size where the focal spot size is projected onto the analyzer grating plane and normalized by the G2 periodicity. The solid line denotes the grating combination where both gratings are geometrically ideal (Table I first row and first column). The dashed line corresponds to the combination where the phase grating layout is geometrically ideal with stabilization gaps and the analyzer grating real (Table I last row and second column). The + represents a measurement with comparable gratings.

The objective function is the visibility

$$
V=\frac{I_{\max }-I_{\min }}{I_{\max }+I_{\min }}
$$

where $I_{\max }$ and $I_{\min }$ denote the maximum and minimum intensity value of an intensity oscillation during the phasestepping [7] observed by a detector pixel. The visibility expresses the contrast of the intensity modulation. The motivation for the visibility is the relation to the error of the reconstructed differential phase where the error is proportional to the reciprocal of the visibility. Therefore, a maximization of the visibility leads to a smaller reconstruction error.

Table I summarizes the results of the simulations. It displays different grating layout combinations. The columns correspond to the used phase grating G1 and the rows to analyzer grating G2 layouts. Additionally, simulations with phase grating using a deformed real analyzer grating layout were performed. A real phase grating layout is comparable to the layout in fig. $3 \mathrm{~b}$.

In an ideal case, where both gratings are geometrically ideal, the visibility corresponds to 0.43 . This deviation from an ideal visibility of 1.00 is mainly caused by gold absorption and blurring of the fringes due to the polychromaticity of the spectrum. Using an ideal phase but a real analyzer grating the visibility drops by $40 \%$ to 0.26 . Adding stabilization gaps in the phase grating layout the visibility drops by further $10 \%$ to 0.23 . Table I rows $2-4$ show also that the position of the gaps do not have any influence to the visibility as long as the area covered by the gaps does not change. The last column emphasizes the required quality regarding the phase grating layout. Using a deformed layout the visibility drops by about $70 \%$.
Fig. 4 displays a plot of visibilities with respect to the normalized projected focal spot size $\left(\sigma^{*}\right)$ where the focal spot size is projected onto the analyzer grating plane and normalized by the G2 periodicity. The solid line represents the visibilities of the grating combination of Table I first row and first column. The dashed line corresponds to the visibilities of Table I last row and second column. Both lines show the loss of visibility while increasing $\sigma^{*}$. Measured visibility with comparable gratings and $\sigma^{*}=0.2$ equals to 0.08 and the simulated to 0.11 .

\section{CONCLUSiON}

This work shows the advantages of the presented simulation framework. It enables the investigation of the influence of the grating layout onto the signal. Furthermore, the results of the simulations emphasize the required quality of the phase grating which has to be as geometrically ideal as possible due to a higher sensitivity. Further, they show that the visibility is independent of the gap positions. All in all, this simulation framework makes the assessment of gratings with respect to their layout easier, faster and cheaper.

\section{ACKNOWLEDGMENTS}

This work was funded by the German ministry of education and science (BMBF 01 EZ 0923 DLR).

\section{REFERENCES}

[1] F. Pfeiffer, T. Weitkamp, O. Bunk, C. David, "Phase retrieval and differential phase-contrast imaging with low-brilliance X-ray sources," Nature Phys. 2, 258-261 (2006).

[2] A. Momose, W. Yashiro, Y. Takeda, Y. Suzuki, T. Hattori, "Phase tomography by X-ray Talbot interferometry for biological imaging," Japanese Journal of Applied Physics, 45 (6A), 5254-5262 (2006).

[3] E. Reznikova, J. Mohr, M. Boehrner, V. Nazmov and P-J. Jakobs, "Soft X-ray lithography of high aspect ratio SU8 submicron structures", Journal of Microsystem Technologies, 1683-1688, (2008)

[4] S.W. Wilkins, T.E. Gureyev, D. Gao, A. Pogany, A.W. Stevenson, "Phasecontrast imaging using polychromatic hard X-rays", Nature 384, 335-337 (1996).

[5] D. M. Paganin, "Coherent X-ray optics" Oxford University Press, Oxford series on synchrotron radiation, (2006)

[6] P. Bartl, J. Durst, W. Haas, T. Michel, A. Ritter, T. Weber, G. Anton, "Simulation of X-Ray Phase-Contrast Imaging Using Grating-Interferometry", Nuclear Science Symposium Conference Record, (2009)

[7] T. Weitkamp, A. Diaz, C. David, F. Pfeiffer, M. Stampanoni, P. Cloetens, E. Ziegler, "X-ray phase imaging with a grating interferometer", Opt. Express, vol. 13, pp. 6296-6304, (2005) 